\title{
Whole genome sequence analysis indicates recent diversification of mammal- associated Campylobacter fetus and implicates a genetic factor associated with $\mathrm{H}_{2} \mathrm{~S}$ production
}

Linda van der Graaf-van Bloois ${ }^{1,2}$, Birgitta Duim ${ }^{1,2}$, William G. Miller ${ }^{3}$, Ken J. Forbes ${ }^{4}$, Jaap A. Wagenaar ${ }^{1,2,5}$ and Aldert Zomer ${ }^{1,2^{*}}$

\begin{abstract}
Background: Campylobacter fetus (C. fetus) can cause disease in both humans and animals. C. fetus has been divided into three subspecies: C. fetus subsp. fetus (Cff), C. fetus subsp. venerealis (Cfv) and C. fetus subsp. testudinum (Cft). Subspecies identification of mammal-associated C. fetus strains is crucial in the control of Bovine Genital Campylobacteriosis (BGC), a syndrome associated with Cfv. The prescribed methods for subspecies identification of the $\mathrm{Cff}$ and $\mathrm{Cfv}$ isolates are: tolerance to $1 \%$ glycine and $\mathrm{H}_{2} \mathrm{~S}$ production.

Results: In this study, we observed the deletion of a putative cysteine transporter in the Cfv strains, which are not able to produce $\mathrm{H}_{2} \mathrm{~S}$ from L-cysteine. Phylogenetic reconstruction of the core genome single nucleotide polymorphisms (SNPs) within Cff and Cfv strains divided these strains into five different clades and showed that the Cfv clade and a Cff clade evolved from a single Cff ancestor.

Conclusions: Multiple C. fetus clades were observed, which were not consistent with the biochemical differentiation of the strains. This suggests the need for a closer evaluation of the current $C$. fetus subspecies differentiation, considering that the phenotypic differentiation is still applied in BGC control programs.

Keywords: Campylobacter fetus, Bovine Genital Campylobacteriosis, Subspecies differentiation, Core genome SNP analysis, $\mathrm{H}_{2} \mathrm{~S}$ production, Cysteine transporter

Abbreviations: BGC, Bovine Genital Campylobacteriosis; C. fetus, Campylobacter fetus; Cff, C. fetus fetus; Cft, C. fetus testudinum; Cfv, C. fetus venerealis; Cfvi, C. fetus venerealis biovar intermedius; Gl, Genomic island; Kya, Thousand (Kilo) years ago; SNP, Single nucleotide polymorphism; T4SS, Type IV secretion system
\end{abstract}

\footnotetext{
* Correspondence: A.L.Zomer@uu.nl

'Department of Infectious Diseases and Immunology, Faculty of Veterinary

Medicine, Utrecht University, Utrecht, The Netherlands

${ }^{2}$ WHO Collaborating Centre for Campylobacter/OIE Reference Laboratory for

Campylobacteriosis, Utrecht, The Netherlands

Full list of author information is available at the end of the article
} 


\section{Background}

The pathogen Campylobacter fetus (C. fetus) can cause disease in both animals and humans. In humans, C. fetus infections vary from acute diarrhea to systemic illness [1]. In animals, C. fetus infections can cause abortion and infertility, mainly in cattle and in sheep [2]. The mammal-associated C. fetus subspecies are C. fetus subsp. fetus (Cff) and C. fetus subsp. venerealis (Cfv) [3], whereas C. fetus subsp. testudinum (Cft) is associated with reptiles [4]. C. fetus subsp. venerealis includes a biochemical variant, designated $C$. fetus subsp. venerealis biovar intermedius (Cfvi) [5].

C. fetus subsp. venerealis has been described to be the causative agent of Bovine Genital Campylobacteriosis (BGC), associated with infertility and abortion in cattle [6]. BGC is notifiable to the World Organisation for Animal Health (OIE). A crucial element in the BGC control program relies on the subspecies identification of $C$. fetus isolates. Currently, the methods prescribed by the OIE to differentiate Cff, Cfv and Cfvi are tolerance to $1 \%$ glycine and $\mathrm{H}_{2} \mathrm{~S}$ production [7]: Cff is tolerant to $1 \%$ glycine and able to produce $\mathrm{H}_{2} \mathrm{~S}$, Cfv is not tolerant to $1 \%$ glycine and not able to produce $\mathrm{H}_{2} \mathrm{~S}$ and Cfvi is not tolerant to $1 \%$ glycine (like $\mathrm{Cfv}$ ) and able to produce $\mathrm{H}_{2} \mathrm{~S}$ (like Cff). The biochemical tests are hampered by poor reproducibility [8], and the phenotypes are not completely consistent with the genomic characteristics of the C. fetus strains, since phenotypically-identified Cff strains were genotypically identical with $\mathrm{Cfv}$ strains [9]. An obvious distinguishing and important feature of $C$. fetus cells is the surface layer (S-layer), which is considered to be associated with the pathogenicity of $C$. fetus strains [10]. C. fetus cells can express two types of surface array proteins (Sap), which correlates with the serotypes of the bacterium; Cfv strains are serotype A; Cff strains may be either serotype $A$ or serotype $B$ and rarely serotype $A B$; and $C f t$ strains are serotype $A$, serotype $B$ or serotype $A B[11,12]$. The molecular method multilocus sequence typing (MLST) was recommended to differentiate $\mathrm{Cff}$ and $\mathrm{Cfv}$ strains [8]. However, a recent study showed that the current MLST scheme was not able to reliably differentiate the $C$. fetus subspecies, as a Cff strain was isolated with the Cfv-associated MLST ST-4 genotype [13].

Whole-genome analysis provides fine-scale resolution of bacterial genomes and allows the calculation of evolutionary events, as shown for C. coli and C. jejuni [14]. Whole-genome analysis has improved, and will continue to improve our understanding of the features that distinguish $C$. fetus subspecies and the evolutionary forces that have acted on C. fetus over time. In this study, we performed a core genome single-nucleotide polymorphisms (SNPs) analysis of 42 C. fetus Cff and Cfv genomes to identify subspecies-specific SNPs. We performed a SNP- based phylogenetic analysis of the core genomes and a BEAST analysis to estimate the divergence dates of Cff and $\mathrm{Cfv}$ strains. Additionally, we investigated whether the genomes contain specific SNPs or genes that could be associated with the biochemical tests and different clinical features of the C. fetus strains.

\section{Methods}

\section{Bacterial strains and whole genome sequencing}

In this study, 42 C. fetus strains from different countries and sources were included (Table 1). The strains were biochemically characterized (except the NCBI GenBank strains $\mathrm{H1}-\mathrm{UY}, 642-21$, B6, and 99/541), using $\mathrm{H}_{2} \mathrm{~S}$ production in medium amended with $0.02 \%$ cysteine- $\mathrm{HCl}$ and $1 \%$ glycine tolerance, as described before [15]. Genotypic subspecies characterisation was performed using MLST [8] and AFLP [16]. The whole genome sequence data of all $C$. fetus genomes was published previously $[9,12,17]$. Briefly, the C. fetus genomes (except the genomes with accession numbers starting with ERR and the genomes downloaded from NCBI GenBank) were sequenced using a Roche 454 GS-FLX+ Genome sequencer with Titanium chemistry and assembled into contigs using the Newbler Assembler (version 2.6). The genomes with accession numbers starting with ERR were sequenced using an Illumina Hiseq and assembled into contigs with the Velvet assembler in an established pipeline at the Sanger Institute. The genomes of four $C$. fetus strains $(04 / 554,97 / 608,03 / 293$ and 01/165) were closed by using a PacBio RS sequencer and assembled into contigs using Quiver (Pacific Biosciences, CA, USA) with the base calls validated using Illumina MiSeq reads.

The sequences with accession numbers starting with ERR are available from the European Nucleotide Archive (ENA) [18], and the remaining sequences are available from NCBI GenBank (Table 1). The following genomes were also included; Cff strain H1-UY (GenBank accession number JYCP00000000), Cfv strain 642-21 (GenBank accession number AJSG00000000), Cfv strain B6 (GenBank accession number AJMC00000000), Cfv strain NCTC 10354 (GenBank accession number AFGH00000000) and Cfvi strain 99/541 (GenBank accession number ASTK00000000).

The quality of the whole genome sequence data was assessed with the Checkm tool [19], showing a suitable completeness score of $>96 \%$ with 600 tested marker genes.

\section{Genome alignment and phylogenetic core genome SNP analysis}

For phylogenetic core genome SNP analysis, whole genome sequences of $42 \mathrm{C}$. fetus isolates were aligned using Parsnp v1.2 [20]. We included Cft strain 03-427 as an outgroup for the phylogenetic core genome SNP analysis, but 
Table 1 Strain characteristics

\begin{tabular}{|c|c|c|c|c|c|c|c|c|c|}
\hline \multirow[t]{2}{*}{ Strain number } & \multirow[t]{2}{*}{ Accession number } & \multirow[t]{2}{*}{ Sero-type } & \multirow[t]{2}{*}{$\begin{array}{l}\text { Bovine } \\
\text { abortion }\end{array}$} & \multirow[t]{2}{*}{$\begin{array}{l}\text { Isolation } \\
\text { year }\end{array}$} & \multirow[t]{2}{*}{$\begin{array}{l}\text { Clade } \\
\text { (Fig. 1) }\end{array}$} & \multicolumn{2}{|l|}{ Subspecies } & \multirow[t]{2}{*}{$\begin{array}{l}1 \% \text { glycine } \\
\text { tolerance }\end{array}$} & \multirow[t]{2}{*}{$\begin{array}{l}\mathrm{H}_{2} \mathrm{~S} \\
\text { production }\end{array}$} \\
\hline & & & & & & Genotype & Phenotype & & \\
\hline $98 / v 445$ & LMBH00000000 & B & No & 1998 & 1 & Cff & $\mathrm{Cff}$ & + & + \\
\hline B0066 & ERR419610 & B & No & 2013 & 1 & Cff & Cff & + & + \\
\hline B0130 & ERR419638 & B & No & 2013 & 1 & $\mathrm{Cff}$ & Cff & + & + \\
\hline B0129 & ERR419637 & B & No & 2013 & 1 & $\mathrm{Cff}$ & $\mathrm{Cff}$ & + & + \\
\hline S0478D & ERR419653 & B & No & 2011 & 1 & $\mathrm{Cff}$ & $\mathrm{Cff}$ & + & + \\
\hline B0042 & ERR419595 & B & No & 2013 & 2 & Cff & Cff & + & + \\
\hline S0693A & ERR419284 & B & No & 2012 & 2 & $\mathrm{Cff}$ & Cff & + & + \\
\hline B0167 & ERR460866 & B & No & 2013 & 2 & Cff & $\mathrm{Cff}$ & + & + \\
\hline B0168 & ERR460867 & B & No & 2013 & 2 & $\mathrm{Cff}$ & $\mathrm{Cff}$ & + & + \\
\hline $04 / 554$ & СР008808-СР008809 & B & Yes & 2004 & 2 & $\mathrm{Cff}$ & Cff & + & + \\
\hline B0047 & ERR419600 & B & No & 2013 & 2 & $\mathrm{Cff}$ & Cff & + & + \\
\hline B0151 & ERR419648 & B & No & 2013 & 2 & Cff & $\mathrm{Cff}$ & + & + \\
\hline B0152 & ERR419649 & B & No & 2013 & 2 & $\mathrm{Cff}$ & Cff & + & + \\
\hline $110800-21-2$ & LSZN00000000 & A & No & 2000 & 3 & $\mathrm{Cff}$ & Cff & + & + \\
\hline B0097 & ERR419623 & A & No & 2013 & 3 & $\mathrm{Cff}$ & Cff & + & + \\
\hline BT 10/98 & LRAL00000000 & A & Unknown & 1998 & 3 & $\mathrm{Cff}$ & $\mathrm{Cff}$ & + & + \\
\hline H1-UY & JYCP00000000 & A & No & 2013 & 4 & $\mathrm{Cff}$ & n.a. & n.a. & n.a. \\
\hline $82-40$ & СР000487 & A & No & 1982 & 4 & $\mathrm{Cff}$ & Cff & + & + \\
\hline B0131 & ERR419639 & A & No & 2013 & 4 & Cff & Cff & + & + \\
\hline Zaf 3 & LREZO00000000 & A & Yes & 2006 & 5 & Cfvi & Cfvi & - & + \\
\hline CCUG 33872 & LREU00000000 & A & Unknown & 1995 & 5 & Cfvi & Cfvi & - & + \\
\hline $642-21$ & AJSG00000000 & A & Unknown & n.a. & 5 & Cfvi & n.a. & n.a. & n.a. \\
\hline ADRI 513 & LRFA00000000 & A & Unknown & n.a. & 5 & $C f v$ & Cfvi & - & + \\
\hline CCUG 33900 & LREV00000000 & A & Yes & 1995 & 5 & $\mathrm{Cfv}$ & $\mathrm{Cfv}$ & - & - \\
\hline LMG 6570 & LREW00000000 & A & Unknown & 1953 & 5 & $\mathrm{Cfv}$ & $\mathrm{Cfv}$ & - & - \\
\hline B6 & AJMC00000000 & A & Unknown & n.a. & 5 & $C f v$ & n.a. & n.a. & n.a. \\
\hline NCTC 10354 & AFGH00000000 & A & Unknown & 1962 & 5 & $C f v$ & $\mathrm{Cfv}$ & - & - \\
\hline 84-112 & HG004426-HG004427 & A & Unknown & 1984 & 5 & $C f v$ & $\mathrm{Cfv}$ & - & - \\
\hline 97/608 & СР008810-СР008812 & A & Yes & 1997 & 5 & $C f v$ & Cfv & - & - \\
\hline B10 & LRET00000000 & A & Unknown & n.a. & 5 & $C f v$ & $\mathrm{Cfv}$ & - & - \\
\hline WBT 011/09 & LMBI00000000 & A & Unknown & 2009 & 5 & Cfvi & Cfvi & - & + \\
\hline Zaf 65 & LREY00000000 & A & Unknown & 2007 & 5 & Cfvi & $\mathrm{Cff}$ & + & + \\
\hline $97 / 532$ & LRER00000000 & A & No & 1997 & 5 & Cfvi & Cfvi & - & + \\
\hline $01 / 165$ & СР014568-СР014570 & $A$ & No & 2001 & 5 & Cfvi & Cfvi & - & + \\
\hline 03/293 & СР0006999-СР007002 & A & Yes & 2003 & 5 & Cfvi & $\mathrm{Cff}$ & + & + \\
\hline 92/203 & LRVL00000000 & A & Yes & 1992 & 5 & Cfvi & Cfvi & - & + \\
\hline 03/596 & LRAM00000000 & $A$ & Yes & 2003 & 5 & Cfvi & Cfvi & - & + \\
\hline $02 / 298$ & LRVK00000000 & $A$ & Yes & 2002 & 5 & Cfvi & Cfvi & - & + \\
\hline ADRI 1362 & LREX00000000 & A & Unknown & 1989 & 5 & Cfvi & $\mathrm{Cff}$ & + & + \\
\hline $98 / 25$ & LRES00000000 & A & Yes & 1998 & 5 & Cfvi & $\mathrm{Cfv}$ & - & - \\
\hline INTA 99/541 & ASTK00000000 & A & Unknown & 1999 & 5 & Cfvi & n.a. & n.a. & n.a. \\
\hline $03-427$ & CP006833 & A & No & 2003 & n.a. & $\mathrm{Cft}$ & $\mathrm{Cft}$ & + & + \\
\hline
\end{tabular}

n.a., not available; + positive; - negative; Cff, C. fetus subsp. fetus; Cfv, C. fetus subsp. venerealis; Cfvi, C. fetus subsp. venerealis biovar intermedius; Cft, C. fetus subsp. testudinum 
excluded this strain from visualization of the phylogenetic core genome SNP tree to get a better resolution of the Cff and Cfv branches. SNP discovery was focused on a comparison between Cff and Cfv strains; therefore, Cft strain 03-427 was replaced by Cff strain 82-40 as a reference for this analysis. Recombination regions in the core genome alignment were detected and visualized using Gubbins [21]. A phylogenetic tree was constructed using FastTree2 [22] with a generalized time-reversible model and gamma correction on the recombination-filtered SNPs in the core genome of all isolates, including $\mathrm{Cft}$ strain 03-427. The resulting tree was rooted on $\mathrm{Cft}$ strain 03-427 using Dendroscope [23] prior to visualization using iTOL [24].

\section{BEAST analysis}

Recombination-filtered non-synonymous SNPs of the mammal-associated C. fetus isolates were extracted from the Gubbins results and used for divergence dating in BEAST [25], using the isolation dates as tip dates in the phylogenetic tree, as outlined in the BEAST manual with the following modifications: $10,000,000 \times$ sampling and a general time reversible (GTR) model plus gamma correction as the distance model. Combinations of a strict clock, log-normal clock, exponential clock and random local clock as the clock model and a constant population, exponentially-growing population, and a Bayesian skyline plot with six groups as demographic models were used. Maximum ESS values and lowest $95 \%$ confidence intervals $(\mathrm{CI})$ in the predicted divergence dates of the clades were obtained with a random local clock and a Bayesian skyline plot with four groups as the demographic model.

\section{Calculation of branch-specific $\mathrm{dN} / \mathrm{dS}$ ratios}

To calculate the $\mathrm{dN} / \mathrm{dS}$ ratio per branch in the phylogenetic tree, we aligned the genomes of the mammal-associated C. fetus isolates with Parsnp v1.2 [20] and Cft strain 03427 as outgroup. Synonymous and non-synonymous SNPs were determined on the basis of their location in the coding regions of the Cff strain 82-40 reference genome. Recombination regions were detected using Gubbins [21] and excluded from the alignment. Ancestral state reconstruction of the node sequences was performed using FastML [26] with a generalized time-reversible model and gamma correction. The $\mathrm{dN} / \mathrm{dS}$ ratios were determined per branch between node sequences.

\section{Comparison of SNPs and genes with phenotypic characteristics of the strains}

As traditional differentiation of $C$. fetus subspecies are based on the biochemical $1 \%$ glycine tolerance and $\mathrm{H}_{2} \mathrm{~S}$ production tests, the genomes were screened for genes and SNPs that were associated with these phenotypic characteristics, and tested for $1 \%$ glycine tolerance and
$\mathrm{H}_{2} \mathrm{~S}$ production in cysteine-rich medium as described above. The protein-encoding gene presence and absence were determined using BLAST-based all vs all comparisons with Prokka-annotated genomes [22] using Roary [27], which clustered the proteins using MCL-edge [28]. A Fisher's exact test was used to calculate the two-tail probability value $(p)$ of respectively the detected SNPs and genes versus the outcome of the biochemical tests.

\section{Calculation of clinical association}

A Fisher's exact test was used to calculate the two-tail probability value $(p)$ of SNPs and genes which were specifically found in strains that were isolated from bovine abortions. Significantly-associated SNPs or genes were checked for their presence in Cff strain 04/554, as this is a non-Cfv bovine clinical strain. SNPs and genes that were not present in this strain were excluded from this analysis, to separate clinical-associated from phylogeneticallyassociated SNPs and genes.

\section{Availability of data}

Genome sequences are available from the European Nucleotide Archive (ENA) and from NCBI GenBank, with the accession numbers listed in Table 1.

\section{Results}

\section{Phylogenetic analysis of the core genome SNPs}

Phylogenetic analysis, based on core genome SNPs using $\mathrm{Cft}$ as the outgroup, showed that the mammalassociated $\mathrm{Cff}$ and $\mathrm{Cfv}$ genomes group into five distinct clades (Fig. 1). The division of clades was consistent with the serotypes of the strains; clades 1 and 2 consist of serotype B strains and clades 3,4 and 5 consist of serotype A strains. The division of clades was also consistent with the classification of MLST sequence types (STs), except for Cff strain H1-UY, which has the Cfv-associated MLST ST-4 genotype [13]. Interestingly, SNP phylogeny showed the divergence of clade 4 and clade 5 from a common Cff ancestor. Clade 5 consists of phenotypically-identified Cff, Cfvi and Cfv strains (Table 1) without a clear separation in the phylogeny. However, when the genotypic characterization is used $[8,16]$, clade 5 consists exclusively of $\mathrm{Cfv}$ and $\mathrm{Cfvi}$ strains and clade 1-4 of Cff strains.

\section{BEAST analysis determined that diversification of the mammal-associated C. fetus is a relatively recent event under disruptive selection pressure}

We used BEAST to determine the divergence date of the different clades (Fig. 2). The topology of the BEAST tree was slightly different than the maximum likelihood (ML) SNPs tree (Fig. 1), especially for the serotype B strains. This may be either the result of using only synonymous SNPs in the BEAST analysis or because of the 


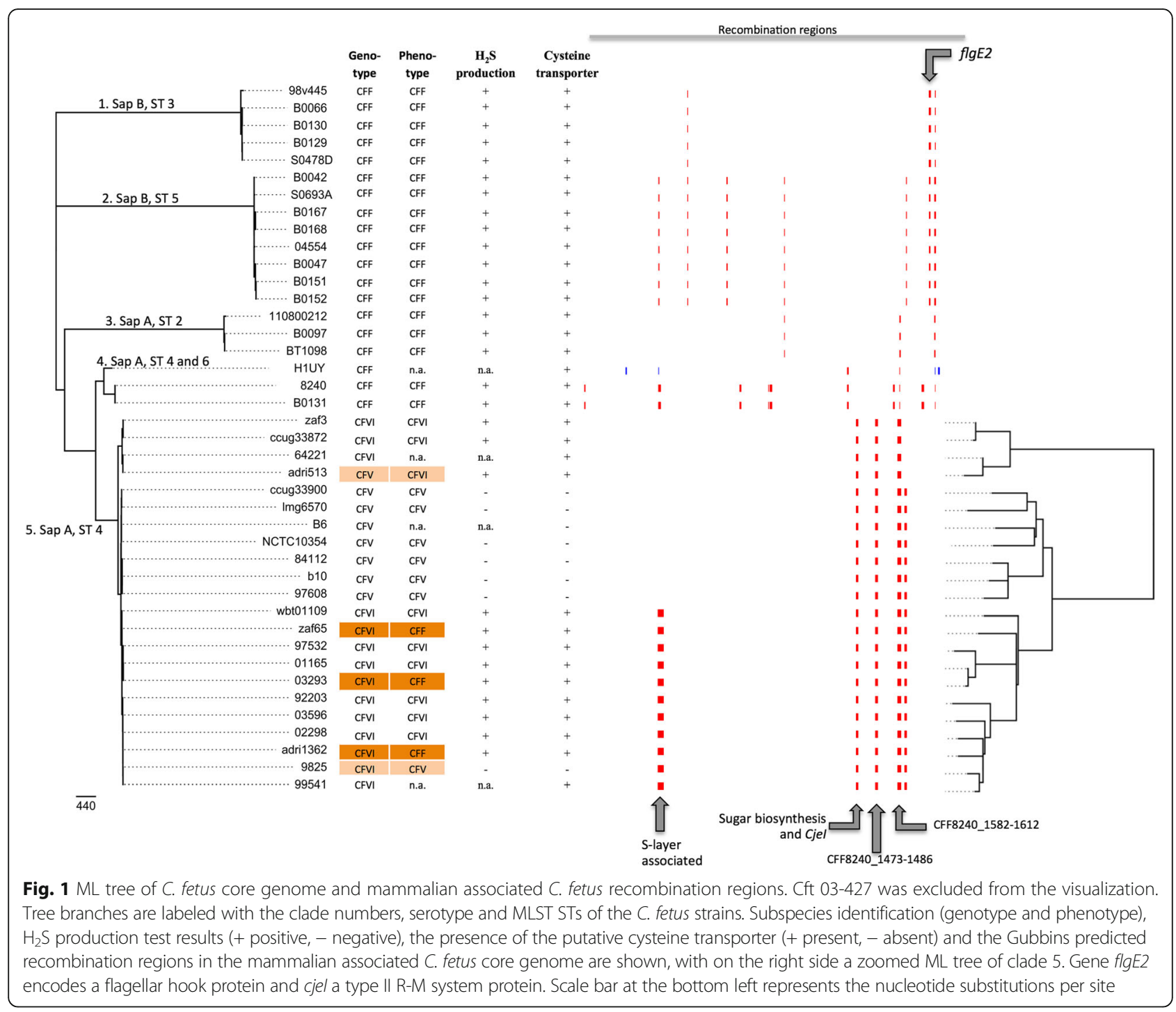

differences between ML methods and methods based on coalescence. The $95 \%$ confidence interval $(\mathrm{CI})$ of the BEAST analysis was too large to estimate the divergence date of clade 2 and clades 3, 4 and 5 [95 \% CI 2.3 Kya $84.9 \mathrm{Kya}]$ and the divergence date of clades 4 and 5 [95 \% CI 0.7 Kya - 19.5 Kya] (Fig. 2). Interestingly, the BEAST analysis showed that all currently circulating strains in clades 1, 2, 3 and 5 diverged very recently from each other.

$\mathrm{Cfv}$ is restricted to the bovine genital tract, but Cff can be isolated from different animal species, e.g. cattle, sheep, goats, pigs, horses, and humans [2]. Therefore, we attempted to investigate whether this change in niche preference has resulted in increased evolutionary pressure on Cfv. We estimated the rate of molecular evolution by comparing the ratio of non-synonymous $(\mathrm{dN})$ versus synonymous (dS) SNPs (Fig. 2). The dN/dS ratio was the highest in the strains of clade $5(0.40)$ compared to the ratio of the strains of clade $4(0.29)$ and in all branches preceding, based on node sequences reconstructed using FastML (Fig. 2). An increased dN/dS ratio $(>0.2)$ is indicative for recently diversifying genomes, whereas lower $\mathrm{dN} / \mathrm{dS}$ ratios $(0.04-0.20)$ correspond to older SNPs and more diverged genomes [29]. All clades have a $\mathrm{dN} / \mathrm{dS}$ ratio $>0.2$, with the highest $\mathrm{dN} / \mathrm{dS}$ ratio of clade 5 of 0.40 , showing that the strains of clade 5 are evolving under relatively more diversifying selection than the strains of the other clades. The estimated molecular clock rate for the mammal-associated $C$. fetus strains was $1.5-3.5 \times 10^{-2}$ substitutions per kb per year, which corresponds with the estimated molecular clock rate of C. jejuni of $1.86-5.81 \times 10^{-2}$ per kb/year [30].

\section{Clade-specific SNPs and orthologs}

The numbers of SNPs and orthologous genes (orthologs) specifically present in each clade (Fig. 1) are presented 


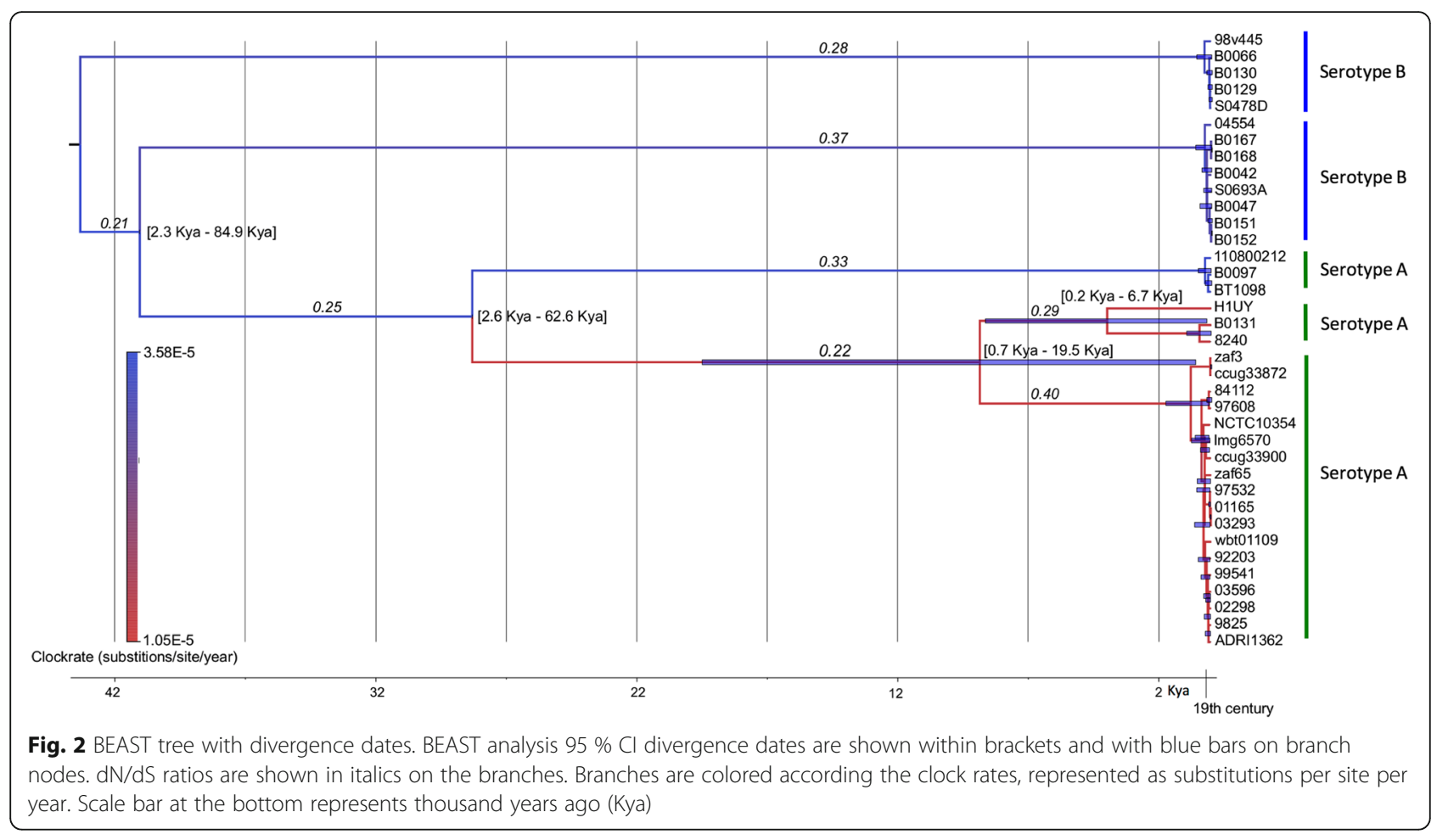

in Table 2, as well as the specific SNPs and orthologs for clades representing the serotypes and subspecies of the strains. The details and annotation of the detected SNPs and orthologs are listed in Additional file 1: Table S1.

Clades 1-2 consisted only of Cff serotype B strains. For clade 1, 1547 SNPs and 2 orthologs were found, specifically present in clade 1 strains (Table 2, Additional file 1: Table S1). The two clade 1-specific orthologs were hypothetical proteins with unknown function. Specific for clade 2, 1703 SNPs and 41 orthologs were found

Table 2 Core genome SNPs specific for C. fetus clades

\begin{tabular}{|c|c|c|c|}
\hline Clade & Representing & $\begin{array}{l}\text { Number of clade- } \\
\text { specific SNPs }\end{array}$ & $\begin{array}{l}\text { Number of clade-specific } \\
\text { orthologs (annotation) }\end{array}$ \\
\hline 1 & Cff serotype B & 1547 & 2 (hypothetical) \\
\hline 2 & Cff serotype B & 1703 & $\begin{array}{l}41 \text { (fic, transposase, } \\
\text { hypothetical) }\end{array}$ \\
\hline 3 & Cff serotype A & 1430 & $\begin{array}{l}6 \text { (R-M system type I } \\
\text { and III) }\end{array}$ \\
\hline 4 & Cff serotype A & 121 & 0 \\
\hline 5 & Cfv and Cfvi & 282 & 0 \\
\hline 5 & $\mathrm{Cfv}$ & 5 & 6 \\
\hline $1-2$ & Cff serotype B, total & 571 & $\begin{array}{l}14 \text { (toxin/antitoxin, R-M, } \\
\text { S-layer associated) }\end{array}$ \\
\hline $3-4$ & Cff serotype A, total & 83 & $\begin{array}{l}14 \text { (glycosyltransferase, } \\
\text { methyltransferase, } \\
\text { ketoreductase) }\end{array}$ \\
\hline
\end{tabular}

Cff, C. fetus subsp. fetus; Cfv, C. fetus subsp. venerealis; Cfvi, C. fetus subsp. venerealis biovar intermedius
(Table 2, Additional file 1: Table S1). The specific orthologs were located in a region encoding mainly hypothetical proteins, as well as a Fic domain protein and a transposase. This clade 2 -specific region is chromosomally located in Cff strain 04/554 (CFF04554_0637 - CFF04554_0684). All strains of both clades 1 and 2 (Cff serotype B) contain 571 specific SNPs and 14 specific orthologs. The clade 1 and 2-specific orthologs included a toxin/antitoxin system (CFF04554_0478 and CFF04554_0479), restrictionmodification (R-M) system-associated genes (CFF04554 1799, CFF04554_0318 and CFF04554_0319) and S-layer associated genes (CFF04554_1746, CFF04554_0481 and CFF04554_0484).

Clades 3 and 4 consist of Cff serotype A strains. Clade 3 contains 1430 specific SNPs and six specific orthologs (Table 2, Additional file 1: Table S1). The specific orthologs encoded hypothetical proteins of unknown function, as well as R-M type I (CFF8240_0988) and R-M type III genes. For clade 4, 121 specific SNPs and no specific orthologs are found. All strains of clades 3-4 (Cff serotype A) contain $83 \mathrm{SNPs}$ and 14 specific orthologs that are mainly located in one region (CFF8240_1591 CFF8240_1608) that includes a glycosyltransferase, a methyltransferase and a ketoreductase.

Clade 5 consists of both genotypically-identified $\mathrm{Cfv}$ and Cfvi strains. For this clade, 282 specific SNPs were found (Additional file 1: Table S1). The genotypicallyidentified $\mathrm{Cfv}$ strains of clade 5 contain five specific SNPs and six specific orthologs. The specific orthologs 
encoded hypothetical proteins, a transcriptional regulator (CFV97608_1300), a resolvase and a transposase.

Analysis of SNP regions that are subject to recombination Interestingly, $42 \%(119 / 282)$ of the SNPs differing between clades $1-4$ and clade 5 are located in regions $<1 \mathrm{~kb}$ apart. This is suggestive of recombination, since on average a SNP would be expected to occur approx. every $6.5 \mathrm{~kb}$, based on the number of SNPs identified here in genomes of approx. $1.85 \mathrm{mbp}$. (1.85 mbp./282 SNPs= $6.5 \mathrm{~kb} / \mathrm{SNP})$. We predicted potential recombination regions in the mammal-associated C. fetus core genome alignment with Gubbins [21] and visualized the results (Fig. 1). Using this analysis, one region showed recombinations in all strains of clades $1-4$ and three recombination regions were found in all strains of clade 5 .

The recombination region common to all strains of clades 1-4 encoded the flagellar hook gene flgE2 (CFF8240_1769) (Fig. 1). All strains of clade 5 contained a recombination region (CFF8240_1393-CFF8240_1398) with a type II R-M gene (cjeI) (CFF8240_1393) and genes involved in sugar biosynthesis, including: a NADdependent epimerase/dehydratase (CFF8240_1396); a nucleotide sugar dehydrogenase (CFF8240_1397); and a polysaccharide biosynthesis protein (CFF8240_1398). Another clade 5-specific recombination region is CFF 8240_1473-CFF8240_1486 that encodes: leader peptidase A (LepA; CFF8240_1473); ribose-phosphate pyrophosphokinase (Prs; CFF8240_1474); a subunit of aspartate carbamoyltransferase (PyrB; CFF8240_1475), a formate dehydrogenase subunit (FdhC; CFF8240_1482) and a glutamate synthase subunit (GltD; CFF8240_1486). In all strains of clade 5 , a recombination region was found (CFF8240_1582-CFF8240_1612), encoding: several radical SAM domain proteins; two transketolase subunits (CFF8240_1587 and CFF8240_1589); a methyltransferase (CFF8240_1590); and two glycosyltransferases (CFF8240_ 1607 and CFF8240_1612). Additionally, the S-layer (sap) region (CFF8240_0455-CFF8240_0501) was also identified by Gubbins as a recombination region, although the sap locus itself is not included in the core genome alignment due to assembly issues of this region [12].

\section{Deletion of a putative cysteine transporter is associated with $\mathrm{H}_{2} \mathrm{~S}$ production-negative strains}

Currently, the OIE-prescribed biochemical tests to differentiate $\mathrm{Cff}, \mathrm{Cfv}$ and $\mathrm{Cfvi}$ consist of determining tolerance to $1 \%$ glycine and production of hydrogen sulfide $\left(\mathrm{H}_{2} \mathrm{~S}\right)$ from L-cysteine [7]. We analyzed the genomes of the C. fetus strains for the presence of specific genes associated with these phenotypic characteristics. Interestingly, $\mathrm{H}_{2} \mathrm{~S}$ negative strains (Table 1 ) did not encode two subunits of an amino-acid $\mathrm{ABC}$ transporter (CFF8240_0780 and CFF8240_0781), that together with an ATP-binding subunit (CFF8240_0779) putatively form an amino-acid $\mathrm{ABC}$ transport system involved in cysteine transport (Kegg module cff_M00234) (Additional file 2). The absence of the two $\mathrm{ABC}$ transporter genes in the $C$. fetus $\mathrm{H}_{2} \mathrm{~S}$ negative strains possibly explains the phenotypic characteristic of these strains. Cfvi strain 98/25 was described before as $\mathrm{H}_{2} \mathrm{~S}$ positive $[8,9]$; however, in this study, the isolates that were sequenced were biochemically characterized and the sequenced isolate of strain 98/25 was $\mathrm{H}_{2} \mathrm{~S}$ production negative, in accordance with the absence of the putative cysteine transporter in this genome.

\section{Conjugative transfer region associated with strains from bovine abortions}

The $C$. fetus strains were arranged according to their clinical features: nine strains were from bovine abortions; seventeen strains were from screenings; and for fifteen strains, the clinical features were unknown (Table 1). No SNPs were found that were both present in the only known Cff strain from a bovine abortion (strain 04/554) and significantly present in the Cfv/Cfvi strains from bovine abortions. However, one region was significantly present in all strains from bovine abortions $(p<0.05)$; this region contains genes encoding a filamentation induced by cyclic AMP (Fic) domain protein (CFV97608_b0017), a DNA-binding protein (CFV97608_b0010) and conjugative transfer (tra) proteins (CFV97608_b00014, CFV97608_ b0015, CFV97608_b0021). This region is located in Cff strain $04 / 554$, Cfv strain $97 / 608$ and Cfv strain $84-112$ on a megaplasmid or an extra-chromosomal element [17]. This tra/trb region is present in 11 C. fetus strains isolated from non-abortion cases; Cff strain 98/v445 and Cfv/Cfvi strains CCUG 33872, 642-21, ADRI 513, B10, 84-112, LMG 6570, NCTC 10354, Zaf 65, ADRI 1362 and 99-541 (Additional file 1).

\section{Discussion}

The core genome SNP analysis of the mammal-associated C. fetus strains identified a large number of SNPs. Since available PCR methods and MLST typing schemes are not able to identify C. fetus strains correctly to subspecies level [13, 15], Cfv/Cfvi-specific SNPs could be used to develop new diagnostic methods, such as a SNP probe-PCR, or to improve the current MLST scheme. However, two drawbacks of a PCR based on Cfv/Cfvi-specific SNPs are that the stability of such SNPs is unknown and that the design of a reliable PCR based on specific SNPs requires a high degree of optimization [31, 32]. In a previous study, we presented the division of the mammal-associated $C$. fetus strains into two different clusters, based on a core gene alignment of five $\mathrm{Cff}$ and $18 \mathrm{Cfv} / \mathrm{Cfvi}$ strains [9]. In this study, $19 \mathrm{Cff}$ strains and $22 \mathrm{Cfv} / \mathrm{Cfvi}$ were included, which gave a better resolution of the branches with Cff strains, when compared to a previous analysis with five Cff strains 
[9]. The current core genome SNP analysis separated the C. fetus strains into five different clades, that contain either the genotypically-identified Cff (four clades) or Cfv/Cfvi (one clade) strains. We demonstrated that the biochemical differentiation of the Cff, Cfv and Cfvi strains is not supported by the core genome SNP phylogeny: clade 5 consists of phenotypically-identified Cff, Cfv and Cfvi strains without a congruent separation in the phylogeny of these strains This showed that the phenotypic separation of the mammal-associated C. fetus strains is not supported by the core genome SNP phylogeny, which gives rise to the consideration if the current phylogenetic subspecies differentiation is still reasonable, as previously mentioned [9].

\section{Forces of Cff and $\mathrm{Cfv}$ diversification}

Phylogenetic analysis indicated that Cfv/Cfvi clade 5 and Cff clade 4 have a common Cff ancestor. No association of the clades with geographic origin and/or host specificity was observed. Interestingly, the short terminal branches and recent diversification suggest that the isolates within each clade, except for strain H1-UY, have diverged recently. This is in contrast to what is observed in $\mathrm{Cft}$, where terminal branches of the isolates are much longer and a much higher genome diversity is observed [11]. The world-wide spread of the mammal-associated C. fetus strains is potentially associated with the improvements in cattle breeding by selection and cross-breeding which started around the 1700s and 1800s, followed by extensive spread of high-producing dairy cows and beef cattle [33], which may have carried only a very limited number of $C$. fetus clones. We suggest that this spread from a limited number of sources has resulted in our current observation of the highly clonal nature of mammal-associated $C$. fetus. Alternatively, the observation of only recently diverged strains is a result of selective sweeps, when a population member with an advantageous trait will take over the population before the trait can spread to other members [34]. It is unlikely that selective sweeps occurred in the mammal-associated $C$. fetus population, since cattle are intensively monitored worldwide for the presence of $C$. fetus subsp. venerealis and only one non-clonal C. fetus strain was found (H1-UY). Cff strain H1-UY was isolated from the blood of a rural worker in 2013, who was diagnosed with cellulitis and was in daily contact with cattle [13].

The rates of changes at non-synonymous and synonymous SNPs indicate whether a gene is under purifying or diversifying selection $[35,36]$ and is expressed in the $\mathrm{dN} / \mathrm{dS}$ ratio. The $\mathrm{dN} / \mathrm{dS}$ ratio of bacterial genes under stabilizing selection falls generally within the range $0.04-0.2$ [37]. In the mammal-associated $C$. fetus, the $\mathrm{dN} / \mathrm{dS}$ ratios were all $>0.2$, showing that all genomes are under diversifying selection. The highest $\mathrm{dN} / \mathrm{dS}$ ratio was found for the clade
5 strains (0.40) (Fig. 2). This high ratio may be due to the genetic features of these strains: they have no CRISPR-cas systems and the clade 5-strains have 24 specific SNPs in the type II R-M system gene cjeI compared to the other strains (Additional file 1: Table S1). Type II R-M systems can undergo major changes in specificity by recombination events as shown for Helicobacter pylori [38] and R$\mathrm{M}$ type II systems may play a role in plasmid transformation, as described for C. jejuni where knockout mutagenesis of gene cjel resulted in a strain with a 1,000-foldenhanced transformation efficiency [39]. It is unknown whether these SNPs influence the functionality or specificity of this type II R-M system in C. fetus strains, but the clade 5-strains are possibly more susceptible to insertion of foreign DNA, since the genomes contain genomic islands (GIs) encoding T4SSs, phages and insertion sequences $[9,17]$.

\section{$\mathrm{H}_{2} \mathrm{~S}$ negative phenotype of $\mathrm{Cfv}$ strains associated with loss of a putative cysteine transporter}

The original classification of the mammal-associated $C$. fetus subspecies Cff and Cfv are based on the $1 \%$ glycine tolerance test [40]. Cfv strains can be discriminated from Cfvi strains with the $\mathrm{H}_{2} \mathrm{~S}$ production test [5]. We show that the $\mathrm{H}_{2} \mathrm{~S}$ negative $C$. fetus strains have lost a putative cysteine transporter. Without the encoded transporter, the cells are possibly less capable of importing cysteine, which under normal situations is reduced while forming $\mathrm{H}_{2} \mathrm{~S}$. The $\mathrm{H}_{2} \mathrm{~S}$-negative $\mathrm{Cfv}$ strains have a niche restriction to the genital tract of cattle, whereas the $\mathrm{H}_{2} \mathrm{~S}$-positive Cfvi and Cff strains are not restricted to the genital tract and assumed to be able to colonize the intestines as well [5]. One may speculate that the Cfv strains have a defect causing the incapability to grow outside of the genital tract, but it is unknown if this partial deletion of the putative cysteine transporter can be associated with the niche restriction of these strains.

\section{Virulence-associated genes specific for clades and strains from bovine abortions}

Since the first description of the $C$. fetus subspecies in 1959 , it is presumed that Cfv cause disease in the genital tract of cows, like enzootic venereal sterility and abortions in pregnant cows, and that Cff only cause sporadic abortions [40]. It is unknown which genomic features are responsible for the pathogenicity of the $C$. fetus strains, but potential candidates are the surface layer of the $C$. fetus cells $[10,41]$ and type IV secretion systems (T4SSs) [17, 42, 43]. We studied the genomes of nine strains from bovine abortions and observed that, in addition to the S-layer proteins and T4SSs, all the strains from bovine abortions contain a region encoding conjugative transfer (tra) proteins. This tra region is located in the closed genomes of strains 04/554, 97/608 
and 84-112 on a plasmid/ICE, which also contain a trbT4SS and fic genes. The fic domain proteins encoded by this region are of potential interest, because fic domain proteins have an immune-modulatory function by influencing the cytoskeleton organization of the host cells [44] and are translocated by T4SSs in Legionella pneumophila, Coxiella burnetii and Bartonella henselae [43], which has also been proposed for C. fetus strains [45]. The trb/tra genes are also present in 11 C. fetus strains isolated from non-abortion cases (Additional file 1). It is possible that these strains can also cause bovine abortions; however, more studies, specifically animal experiments investigating the virulence of $C$. fetus strains are required to assess this hypothesis.

\section{Conclusion}

Phylogenetic core genome SNP analysis divided the mammal-associated C. fetus strains into five different clades, which were consistent with the serotypes, but not with the phenotypes of the strains. BEAST analysis showed that the clade with genotypically-identified $\mathrm{Cfv} /$ Cfvi strains has evolved from a Cff ancestor under diversifying selection. Phylogenetic analysis of the core genome SNPs did not differentiate $\mathrm{H}_{2} \mathrm{~S}$-negative Cfv from $\mathrm{H}_{2} \mathrm{~S}$ positive Cfvi strains. The partial deletion of a putative cysteine transporter is observed in all $\mathrm{H}_{2} \mathrm{~S}$ negative Cfv strains.

\section{Additional files}

Additional file 1: Table S1. Table with details of $C$. fetus SNPs and genes, specific for clades and bovine abortion strains. (XLSX $4167 \mathrm{~kb}$ )

Additional file 2: Figure S1. Schematic representation of the putative cysteine transporter genes CFF8240_0779 - CFF8240_0781 (white) in strain Cff 8240 with flanking genes rarD and psgA (grey). The start and stop positions of respectively rarD and psgA are shown in italics. Cfv strain 97/608 lacks one complete ORF and contain a remnant of one ORF of the putative cysteine transporter. Shown are the similar deletion sites sequences in the Cfv strains containing the incomplete transporter. (PDF $149 \mathrm{~kb}$ )

\section{Acknowledgements}

We like to thank Emma Yee (U.S. Department of Agriculture) for the generation of sequence data, we thank James Bono (U.S. Department of Agriculture) for the generation of PacBio RS reads and thank Dr. Brian Brooks and Dr. John Devenish (Canadian Food Inspection Agency) for providing C. fetus strains and for critical review of this manuscript.

\section{Funding}

Publication charges for this article have been funded by Utrecht University, the Netherlands.

\section{Availability of data and materials}

Sequencing data is available from the European Nucleotide Archive (ENA) and NCBI GenBank under the accession numbers listed in Table 1. Datasets supporting the results of this article are also included in the additional files. The used files for tree generation were uploaded to Treebase repository (Access URL for Fig. 1: http://purl.org/phylo/treebase/phylows/study/ TB2:S19749? $x$-access-code=abdd0503f86f2e57eeec93a478f31 cb1\&format=html and for Fig. 2: http://purl.org/phylo/treebase/phylows/study/TB2:S19751?xaccess-code=1e4d7b80cbcedccf9c1 b0db7c32bdaff\&format=html).

\section{Authors' contributions}

LG, BD, JW, AZ conceived this study. LG, WG, KF, AZ performed the experiments. $L G, B D, J W, A Z$ analyzed the data. $L G$ wrote the manuscript. $B D$, WM, KF, JW, AZ were involved in discussions of the work and manuscript revision. All authors approved the final version.

\section{Competing interests}

The authors declare that they have no competing interests.

\section{Consent for publication}

Not applicable.

\section{Ethics approval and consent to participate}

The bacterial isolates were already part of an anonymized strain collection. Evaluation of human patient data has not been performed, therefore no ethics approval was considered necessary.

\section{Author details}

'Department of Infectious Diseases and Immunology, Faculty of Veterinary Medicine, Utrecht University, Utrecht, The Netherlands. ${ }^{2}$ WHO Collaborating Centre for Campylobacter/OIE Reference Laboratory for Campylobacteriosis, Utrecht, The Netherlands. ${ }^{3}$ U.S. Department of Agriculture, Produce Safety and Microbiology Research Unit, Agricultural Research Service, Albany, CA, USA. ${ }^{4}$ School of Medicine and Dentistry, University of Aberdeen, Aberdeen, UK. ${ }^{5}$ Central Veterinary Institute of Wageningen UR, Lelystad, The Netherlands.

Received: 22 April 2016 Accepted: 30 August 2016

Published online: 06 September 2016

\section{References}

1. Wagenaar JA, van Bergen MA, Blaser MJ, Tauxe RV, Newell DG, van Putten JP. Campylobacter fetus infections in humans: exposure and disease. Clin Infect Dis. 2014:58(11):1579-86.

2. Thompson SA, Blaser MJ. Pathogenesis of Campylobacter fetus. In: Nachamkin I, Szymanski CM, Blaser MJ, editors. Campylobacter. 3rd ed. Washington, DC: ASM Press; 2008. p. 401-27.

3. Smibert RM. Genus II Campylobacter Sebald and Véron 1963, 907. In: Krieg $\mathrm{NR}$, Holt HG, editors. Bergey's manual of systematic bacteriology, vol. 1. Baltimore: Williams, \& Wilkins Co; 1984. p. 111-8.

4. Fitzgerald C, Tu ZC, Patrick M, Stiles T, Lawson AJ, Santovenia M, Gilbert MJ, van Bergen M, Joyce K, Pruckler J, Stroika S, Duim B, Miller WG, Loparev V, Sinnige JC, Fields Pl, Tauxe RV, Blaser MJ, Wagenaar JA. Campylobacter fetus subsp. testudinum subsp. nov., isolated from humans and reptiles. Int I Syst Evol Microbiol. 2014;64(Pt 9):2944-8.

5. Florent A. A propos dese vibrions responsables de la vibriose génitale des bovins et des ovins. Bull Off Int Epizoot. 1963:60:1063-74.

6. Garcia MM, Eaglesome MD, Rigby C. Campylobacters important in veterinary medicine. Vet Bull. 1983:53(9):793-818.

7. OIE. Bovine Genital Campylobacteriosis. In: Anonymous, editor. Manual of Diagnostic Tests and Vaccines for Terrestrial Animals (mammal, birds and bees). 7th ed. Paris: Office International des Epizooties; 2012. p. 652.

8. van Bergen MAP, Dingle KE, Maiden MC, Newell DG, van der Graaf-Van Bloois L, van Putten JP, Wagenaar JA. Clonal nature of Campylobacter fetus as defined by multilocus sequence typing. J Clin Microbiol. 2005;43(12):5888-98.

9. van der Graaf-van Bloois L, Miller WG, Yee E, Rijnsburger M, Wagenaar JA, Duim B. Inconsistency of phenotypic and genomic characteristics of Campylobacter fetus subspecies requires re-evaluation of current diagnostics. J Clin Microbiol. 2014;52(12):4183-8.

10. Blaser MJ, Smith PF, Hopkins JA, Heinzer I, Bryner JH, Wang WL. Pathogenesis of Campylobacter fetus infections: serum resistance associated with high-molecular-weight surface proteins. J Infect Dis. 1987;155(4):696-706.

11. Tu ZC, Dewhirst FE, Blaser MJ. Evidence that the Campylobacter fetus sap locus is an ancient genomic constituent with origins before mammals and reptiles diverged. Infect Immun. 2001;69(4):2237-44.

12. Gillbert MJ, Miller WG, Yee E, Zomer AL, van der Graaf-Van Bloois $L$, Fitzgerald C, Forbes KJ, Meric G, Sheppard SK, Wagenaar JA, Duim B. Comparative genomics of Campylobacter fetus from reptiles and mammals reveals divergent evolution in host-associated lineages. Genome Biol Evol. 2016;8(6):2006-19 
13. Iraola G, Betancor L, Calleros L, Gadea P, Algorta G, Galeano S, Muxi P, Greif G, Perez R. A rural worker infected with a bovine-prevalent genotype of Campylobacter fetus subsp. fetus supports zoonotic transmission and inconsistency of MLST and whole-genome typing. Eur J Clin Microbiol Infect Dis. 2015;34(8):1593-6.

14. Sheppard SK, Maiden MC. The evolution of Campylobacter jejuni and Campylobacter coli. Cold Spring Harb Perspect Biol. 2015;7(8):a018119.

15. van der Graaf-van Bloois L, van Bergen MA, van der Wal FJ, de Boer AG, Duim B, Schmidt T, Wagenaar JA. Evaluation of molecular assays for identification Campylobacter fetus species and subspecies and development of a C. fetus specific real-time PCR assay. J Microbiol Methods. 2013;95(1):93-7.

16. Wagenaar JA, van Bergen MAP, Newell DG, Grogono-Thomas R, Duim B. Comparative study using amplified fragment length polymorphism fingerprinting, PCR genotyping, and phenotyping to differentiate Campylobacter fetus strains isolated from animals. J Clin Microbiol. 2001;39(6):2283-6.

17. van der Graaf-Van Bloois L, Miller WG, Yee E, Gorkiewicz G, Forbes KJ, Zomer AL, Wagenaar JA, Duim B. Campylobacter fetus subspecies contain conserved type IV secretion systems on multiple genomic islands and plasmids. PLoS One. 2016;11(4):e0152832.

18. Leinonen R, Akhtar R, Birney E, Bower L, Cerdeno-Tarraga A, Cheng Y, Cleland I, Faruque N, Goodgame N, Gibson R, Hoad G, Jang M, Pakseresht N, Plaister S, Radhakrishnan R, Reddy K, Sobhany S, Ten Hoopen P, Vaughan R, Zalunin V, Cochrane G. The European Nucleotide Archive. Nucleic Acids Res. 2011;39:D28-31.

19. Parks DH, Imelfort M, Skennerton CT, Hugenholtz P, Tyson GW. CheckM: assessing the quality of microbial genomes recovered from isolates, single cells, and metagenomes. Genome Res. 2015;25(7):1043-55.

20. Treangen TJ, Ondov BD, Koren S, Phillippy AM. The Harvest suite for rapid core-genome alignment and visualization of thousands of intraspecific microbial genomes. Genome Biol. 2014;15(11):524.

21. Croucher NJ, Page AJ, Connor TR, Delaney AJ, Keane JA, Bentley SD, Parkhill J, Harris SR. Rapid phylogenetic analysis of large samples of recombinant bacterial whole genome sequences using Gubbins. Nucleic Acids Res. 2015:43(3):e15.

22. http://meta.microbesonline.org/fasttree/. Accessed 04/12 2016.

23. Huson DH, Scornavacca C. Dendroscope 3: an interactive tool for rooted phylogenetic trees and networks. Syst Biol. 2012;61(6):1061-7.

24. Letunic I, Bork P. Interactive Tree Of Life (iTOL): an online tool for phylogenetic tree display and annotation. Bioinformatics. 2007;23(1):127-8.

25. Drummond AJ, Rambaut A. BEAST: Bayesian evolutionary analysis by sampling trees. BMC Evol Biol. 2007;7:214.

26. Pupko T, Pe'er I, Hasegawa M, Graur D, Friedman N. A branch-and-bound algorithm for the inference of ancestral amino-acid sequences when the replacement rate varies among sites: Application to the evolution of five gene families. Bioinformatics. 2002;18(8):1116-23.

27. Page AJ, Cummins CA, Hunt M, Wong VK, Reuter S, Holden MT, Fookes M, Falush D, Keane JA, Parkhill J. Roary: rapid large-scale prokaryote pan genome analysis. Bioinformatics. 2015;31(22):3691-3.

28. Enright AJ, Van Dongen S, Ouzounis CA. An efficient algorithm for largescale detection of protein families. Nucleic Acids Res. 2002;30(7):1575-84.

29. Castillo-Ramirez S, Harris SR, Holden MT, He M, Parkhill J, Bentley SD, Feil EJ. The impact of recombination on $\mathrm{dN} / \mathrm{dS}$ within recently emerged bacterial clones. PLoS Pathog. 2011;7(7):e1002129.

30. Wilson DJ, Gabriel E, Leatherbarrow AJ, Cheesbrough J, Gee S, Bolton E, Fox A, Hart CA, Diggle PJ, Fearnhead P. Rapid evolution and the importance of recombination to the gastroenteric pathogen Campylobacter jejuni. Mol Biol Evol. 2009;26(2):385-97.

31. Hur D, Kim MS, Song M, Jung J, Park H. Detection of genetic variation using dual-labeled peptide nucleic acid (PNA) probe-based melting point analysis. Biol Proced Online. 2015; 17:14-015-0027-5. eCollection 2015.

32. Syvanen AC. Accessing genetic variation: genotyping single nucleotide polymorphisms. Nat Rev Genet. 2001;2(12):930-42.

33. Orozco-terWengel $P$, Barbato $M$, Nicolazzi $E$, Biscarini $F$, Milanesi $M$, Davies W, Williams D, Stella A, Ajmone-Marsan P, Bruford MW. Revisiting demographic processes in cattle with genome-wide population genetic analysis. Front Genet. 2015;6:191.

34. Bendall ML, Stevens SL, Chan LK, Malfatti S, Schwientek P, Tremblay J, Schackwitz W, Martin J, Pati A, Bushnell B, Froula J, Kang D, Tringe SG, Bertilsson S, Moran MA, Shade A, Newton RJ, McMahon KD, Malmstrom RR. Genome-wide selective sweeps and gene-specific sweeps in natural bacterial populations. ISME J. 2016;10:1589-601.
35. Kimura M. Recent development of the neutral theory viewed from the Wrightian tradition of theoretical population genetics. Proc Natl Acad Sci U S A. 1991;88(14):5969-73.

36. Nei M. Selectionism and neutralism in molecular evolution. Mol Biol Evol. 2005;22(12):2318-42

37. Rocha EP, Smith JM, Hurst LD, Holden MT, Cooper JE, Smith NH, Feil EJ. Comparisons of $\mathrm{dN} / \mathrm{dS}$ are time dependent for closely related bacterial genomes. J Theor Biol. 2006;239(2):226-35.

38. Nobusato A, Uchiyama I, Kobayashi I. Diversity of restriction-modification gene homologues in Helicobacter pylori. Gene. 2000;259(1-2):89-98.

39. Holt JP, Grant AJ, Coward C, Maskell DJ, Quinlan JJ. Identification of Cj1051C as a major determinant for the restriction barrier of Campylobacter jejuni strain NCTC11168. Appl Environ Microbiol. 2012;78(22):7841-8.

40. Florent A: Les deux vibriosis génitales; la vibriose due à $V$. fetus venerealis et la vibriose d'origine intestinale due à $V$. fetus intestinalis. Mededelingen der Veeartsenijschool van de RijksUniversiteit te Gent. 1959;3:1-60.

41. Blaser MJ. Role of the S-layer proteins of Campylobacter fetus in serumresistance and antigenic variation: a model of bacterial pathogenesis. Am J Med Sci. 1993;306(5):325-9.

42. Kienesberger $\mathrm{S}$, Sprenger $\mathrm{H}$, Wolfgruber $\mathrm{S}$, Halwachs B, Thallinger GG, PerezPerez Gl, Blaser MJ, Zechner EL, Gorkiewicz G. Comparative genome analysis of Campylobacter fetus subspecies revealed horizontally acquired genetic elements important for virulence and niche specificity. PLoS One. 2014;9(1):e85491.

43. Gorkiewicz G, Kienesberger S, Schober C, Scheicher SR, Gully C, Zechner R, Zechner EL. A genomic island defines subspecies-specific virulence features of the host-adapted pathogen Campylobacter fetus subsp. venerealis. J Bacteriol. 2010;192(2):502-17.

44. Worby CA, Mattoo S, Kruger RP, Corbeil LB, Koller A, Mendez JC, Zekarias B, Lazar C, Dixon JE. The fic domain: regulation of cell signaling by adenylylation. Mol Cell. 2009;34(1):93-103.

45. Kienesberger S, Schober Trummler C, Fauster A, Lang S, Sprenger H, Gorkiewicz G, Zechner EL. Interbacterial macromolecular transfer by the Campylobacter fetus subsp. venerealis type IV secretion system. J Bacteriol. 2011;193(3):744-58.

\section{Submit your next manuscript to BioMed Central and we will help you at every step:}

- We accept pre-submission inquiries

- Our selector tool helps you to find the most relevant journal

- We provide round the clock customer support

- Convenient online submission

- Thorough peer review

- Inclusion in PubMed and all major indexing services

- Maximum visibility for your research

Submit your manuscript at www.biomedcentral.com/submit
) Biomed Central 